3. Ein Wolfram-Einkristall wird auf $78^{\circ} \mathrm{K}$ gekühlt und mit $\mathrm{Kr}-85$ beschossen (Strahlrichtung in diesen und den folgenden Versuchen wieder $7,5^{\circ}$ aus $\langle 111\rangle$ ). Nach Aufwärmen auf Zimmertemperatur erhalten wir das Reichweiteprofil Kurve 3. Der supertail ist stark ausgeprägt.

4. Ein Wolfram-Einkristall wurde auf $78^{\circ} \mathrm{K}$ gekühlt, mit Kr-85 beschossen und anschließend mit einer hohen Dosis von $50 \mathrm{keV} \cdot \mathrm{He}^{+}$-Ionen bestrahlt (Dosis $\left.1,3 \cdot 10^{16} / \mathrm{cm}^{2}\right)$. Nach dem Aufwärmen auf Zimmertemperatur wurde das Profil Kurve 4 erhalten. Der Anteil der $\mathrm{Kr}$-85-Ionen im supertail hat deutlich abgenommen.

5. Der Versuch 4 wurde mit der 2,5fachen $\mathrm{He}^{+}$-Dosis wiederholt: Kurve 5. Der letzte Meßpunkt bei $7500 \AA$ ist wegen des geringen $\mathrm{Kr}$-85-Gehaltes bereits unsicher; in größeren Tiefen ließ sich kaum noch $\mathrm{Kr}$-85 nachweisen.

Aus diesen Ergebnissen darf geschlossen werden: Der supertail bildet sich bei $78{ }^{\circ} \mathrm{K}$ erst nach der Bestrahlung aus, während der Aufwärmung auf Zimmertemperatur. Er kommt durch die Diffusion von rund $10^{-2 \%}$ der eingeschossenen Kr-Ionen zustande. Aus

\section{Einfluß von paramagnetischen Verunreinigungen auf die kernmagnetischen Relaxationszeiten von adsorbiertem Wasser}

\section{MicheL}

Physikalisches Institut der Universität Leipzig

(Z. Naturforschg. 21 a, 366-368 [1966] ; eingeg. am 12. Februar 1966)

Um den Unterschied zwischen dem gemessenen und dem mit den Formeln der dipolaren Proton-ProtonWechselwirkung zwischen zwei gleichen Spins bei isotroper Bewegung ${ }^{1}$ berechneten Verhältnis $T_{1} / T_{2}$ der kernmagnetischen Protonenrelaxationszeiten des adsorbierten Wassers zu deuten, werden unterschiedliche Annahmen gemacht. Winkler ${ }^{2}$ führt das um eine Größenordnung zu kleine Verhältnis $\left(T_{1} / T_{2}\right)$ expt. (vgl. auch Tab. 2) darauf zurück, daß die longitudinale Relaxationszeit hauptsächlich durch eine Wechselwirkung mit paramagnetischen Verunreinigungen bestimmt wird, während die transversale Relaxation im wesentlichen durch die Proton-Proton-Wechselwirkung verursacht werden soll. Woessner und Zimmerman ${ }^{3}$ erklären dagegen den gleichen Effekt durch eine anisotrope Bewegung der adsorbierten Moleküle bei ausschließlicher Proton-Proton-Wechselwirkung.

Zur Klärung dieser Frage wurden die Protonenrelaxationszeiten an Gemischen aus $\mathrm{H}_{2} \mathrm{O}$ und $\mathrm{D}_{2} \mathrm{O}$, die an verschiedenen Adsorbentien sorbiert sind, in Abhängigkeit vom Mischungsverhältnis, aber bei gleicher Gesamtbeladung gemessen. Dabei handelt es sich um

1 A. Abragam, The Principles of Nuclear Magnetism, Oxford 1961, Kap. VIII.

2 H. WinkLER, Z. Naturforschg. 16 a, 780 [1961].

3 D. E. Woessner u. J. R. Zimmerman, J. Phys. Chem. 67, 1590 [1963]. den Versuchen bei $78{ }^{\circ} \mathrm{K}$ und Zimmertemperatur kann die Aktivierungsenergie der Diffusion abgeschätzt werden. Für $D=D_{0} \cdot \exp \{-Q /(R T)\}$ wird mit $D_{0}=0,1$ $\mathrm{cm}^{2} \mathrm{sec}^{-1}$ (Versuchszeit rund $10^{5} \mathrm{sec}$ ) die Aktivierungsenergie im Intervall $0,3<Q<0,8 \mathrm{eV}$ liegen. Dieser relativ niedrige Wert von $Q$ spricht dafür, daß die diffundierenden $\mathrm{Kr}$-85-Atome sich auf Zwischengitterplätzen befinden. Durch den $\mathrm{He}^{+}$-Beschuß werden Senken erzeugt, in denen bei der nachträglichen Diffusion ein großer Teil der Kr-Atome eingefangen und immobilisiert wird. Die Zunahme des supertail-Anteils bei Einschuß in 〈111〉 (Kurve 2) ist gleichzeitig ein Hinweis, daß die infolge der Lenkung durch die parallel zum Strahl liegenden Atomreihen tief (um $1000 \AA$ ) ins Gitter eindringenden Ionen nach der Abbremsung zumindest teilweise als Zwischengitteratome liegen bleiben.

A nmerkung bei der Korrektur: J. A. Davies (Chalk River, Ontario, Canada) hat durch Variation der Einschußrichtung die isotrope Verteilung der Atome im supertail nachgewiesen. Auch dieser Befund deutet auf eine Diffusionsausbreitung hin. (Private Mitteilung; Veröffentlichung in Phys. Rev. vorgesehen.)

$\mathrm{Al}_{2} \mathrm{O}_{3}$ (wasserfrei reinst, Charge 744843 , paramagnetische Verunreinigungen: $\mathrm{Fe}<0,01 \%$ ) und $\mathrm{SiO}_{2} \mathrm{I}$ (aktiv, standard. nach Spengler, Charge 813514 , paramagnetische Verunreinigungen: $\mathrm{Fe}<0,1 \%, \mathrm{Cu}$ und V spektralanalytisch) der Fa. E. M e r c k, Darmstadt, sowie um ein sehr sauberes, durch Hydrolyse von $\mathrm{SiCl}_{4}$ hergestelltes $\mathrm{SiO}_{2} \mathrm{II}^{4}$. Daten zur Struktur dieser Materialien sind in der Tab. 1 dargestellt.

An der z. B. in ${ }^{5}$ beschriebenen Apparatur wurden die Relaxationszeiten $T_{1}$ aus dem Verlauf der freien Induktion nach einer $\pi / 2-\pi / 2$-Impulsfolge und $T_{2}$ aus dem Abfall einer Echo-Serie nach Carr und PurcELL bestimmt. Bei schlechtem Signal-Rausch-Verhältnis wurde die transversale Relaxationszeit außerdem an einem Spektrometer mit digitaler Anzeige ${ }^{6}$ gemessen.

\begin{tabular}{|l|r|r|l|}
\hline & $S\left[\mathrm{~m}^{2} / \mathrm{g}\right]$ & $V\left[\mathrm{~cm}^{3} / \mathrm{g}\right]$ & Porenradienverteilung \\
\hline $\mathrm{Al}_{2} \mathrm{O}_{3}{ }^{7}$ & 98 & 0,222 & $\begin{array}{l}\text { breites Max. bei } r=30 \AA \\
\text { (Halbwertsbreite } 33 \AA)\end{array}$ \\
$\mathrm{SiO}_{2} \mathrm{I}$ & $\approx 530$ & 0,767 & $\begin{array}{l}\text { Max. bei } 2 r=55 \AA \\
V_{\mathrm{m}} \approx 0,106 \mathrm{~cm}^{3} / \mathrm{g}\end{array}$ \\
$\mathrm{SiO}_{2} \mathrm{II}$ & $\approx 600$ & 0,747 & $\begin{array}{l}\text { Hauptmax. bei } 2 r=80 \AA \\
\text { Nebenmax. bei } 2 r=35 \AA \\
V_{\mathrm{m}} \approx 0,118 \mathrm{~cm}^{3} / \mathrm{g}\end{array}$ \\
\hline
\end{tabular}

Tab. 1. Daten zur Struktur. $S=$ spezifische Oberfläche, $V=\mathrm{Ge}$ samtmikroporenvolumen mit $2 r \lesssim 500 \AA$ (aus $\mathrm{N}_{2}$-Isotherme), $V_{\mathrm{m}}=$ Mikroporenvolumen mit $2 r \lesssim 10 \AA, r=$ Porenradius.

${ }^{4}$ R. K. ILer, The Colloid Chemistry of Silica and the Silicates, Cornell University Press, New York 1955.

5 H. Pfeifer, in A. Lösche u. W. Schürz, Hochfrequenz-Spektroskopie, Akademieverlag, Berlin 1961.

6 D. Fenzke, Phys. Letters 13, 215 [1964].

7 G. Ebert, Dissertation, Leipzig 1959. 
In freien, nicht adsorbierten $\mathrm{H}_{2} \mathrm{O}-\mathrm{D}_{2} \mathrm{O}$-Gemischen wächst die longitudinale Relaxationszeit $T_{1}$ der Protonen mit zunehmender Konzentration $\beta$ von $\mathrm{D}_{2} \mathrm{O}$ in $\mathrm{H}_{2} \mathrm{O}$ gemäß

$$
T_{1}^{-1}=T_{1 W}^{-1} \cdot \eta(\beta-1+\beta R)
$$

an ${ }^{8}$, wobei $T_{1 \mathrm{~W}}$ die Relaxationszeit im reinen Wasser, $\eta$ das Verhältnis der Viskositäten des Gemisches zu der des $\mathrm{H}_{2} \mathrm{O}$ und $R=0,042$ eine Konstante bezeichnet. Bei der transversalen Relaxationszeit sind die Verhältnisse ähnlich, jedoch spielt hier noch die skalare Wechselwirkung zwischen den Protonen und den Deuteronen eine Rolle ${ }^{9}$.

Beispielsweise ergibt sich

$$
\left[T_{2}(\beta=0,75)\right] /\left[T_{2}(\beta=0)\right] \approx 2
$$

und nach Gl. (1)

$$
\left[T_{1}(\beta=0,75)\right] /\left[T_{1}(\beta=0)\right] \approx 3 .
$$

Falls bei den Adsorbentien (vgl. Tab. 1) die Protonenrelaxation durch eine Proton-Proton-Wechselwirkung verursacht wird, erwarten wir eine Vergrößerung der Relaxationszeiten mit wachsendem Wert von $\beta$.

Wenn man annimmt, daß sich die isotope Zusammensetzung in der Hydrathülle eines paramagnetischen Ions nicht von der des freien Wassers unterscheidet und alle für die Wechselwirkung zwischen den Protonen und dem paramagnetischen Ion charakteristischen Korrelationszeiten konzentrationsunabhängig sind, dann sollten die Protonenrelaxationszeiten unabhängig vom Mischungsverhältnis sein (vgl. z. B. SpRINZ ${ }^{10}$ ). Dies wurde experimentell für $\mathrm{Al}_{2} \mathrm{O}_{3}$ und $\mathrm{SiO}_{2} \mathrm{I}$ gefunden.

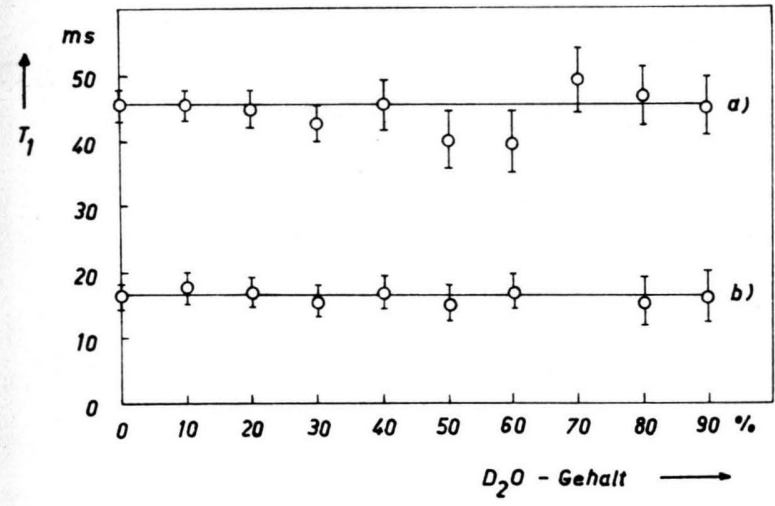

Abb. 1. Abhängigkeit der longitudinalen Protonenrelaxationszeit vom $\mathrm{H}_{2} \mathrm{O}-\mathrm{D}_{2} \mathrm{O}$-Mischungsverhältnis $\left(21^{\circ} \mathrm{C}, \nu=16 \mathrm{MHz}\right)$ a) $150 \mathrm{mg}$ Wasser pro $1 \mathrm{~g} \mathrm{Al}_{2} \mathrm{O}_{3}$, b) $600 \mathrm{mg}$ Wasser pro $1 \mathrm{~g}$ $\mathrm{SiO}_{2}$ I. Das gleiche Verhalten der Relaxationszeiten zeigt sich auch bei anderen Beladungen.

Wie die Abb. 1 und 2 zeigen, bleiben die Relaxationszeiten $T_{1}$ und $T_{2}$ mit zunehmender Verdünnung innerhalb der Fehlergrenzen konstant. Die Unabhängigkeit

8 A. Abragam ${ }^{1}$, S. 328.

9 W. Lohmann, Dissertation, Leipzig 1966.

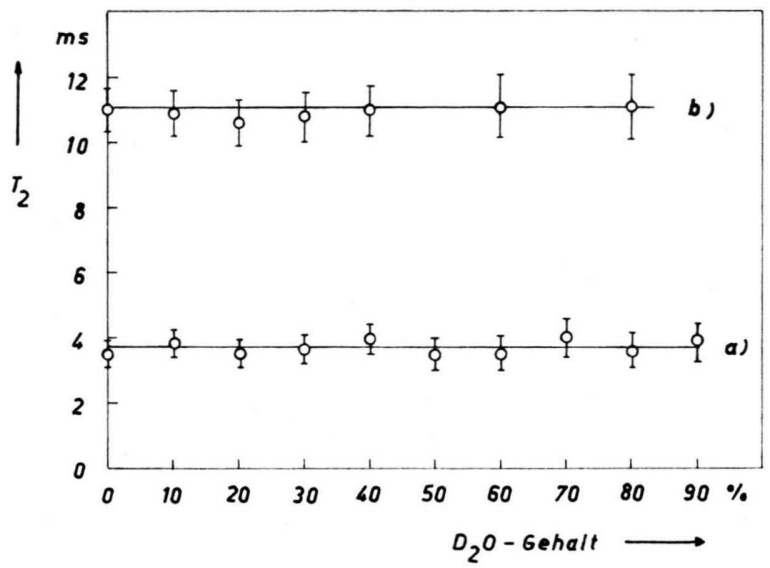

Abb. 2. Abhängigkeit der transversalen Protonenrelaxationszeit vom $\mathrm{H}_{2} \mathrm{O}-\mathrm{D}_{2} \mathrm{O}$-Mischungsverhältnis (Bezeichnungen vgl. Abb. 1).

$\operatorname{der} T_{1}$-Werte vom Mischungsverhältnis stimmt mit den Vorstellungen von $\mathrm{W}_{\text {INKLER }}{ }^{2}$ überein, da $\beta$ die longitudinale Relaxation maßgeblich durch die Wechselwirkung zwischen Protonen und paramagnetischen Ionen bestimmt wird. Dagegen läßt sich das Verhalten von $T_{2}$ weder mit den Vorstellungen von W INKLER $^{2}$ noch von Woessner und Zimmerman ${ }^{3}$ erklären, und man muß annehmen, daß auch $T_{2}$ hauptsächlich durch eine Wechselwirkung mit paramagnetischen Verunreinigungen bestimmt wird. Es gelang aber weder am beladenen noch am unbeladenen Adsorbens, diese paramagnetischen Zentren mit Hilfe eines ESR-Spektrometers hoher Empfindlichkeit (10 $10^{11}$ Spins/Gauß, X-Band) bei Zimmertemperatur nachzuweisen. Dies ist ein Beweis dafür, daß die kernmagnetische Resonanz unter Um-

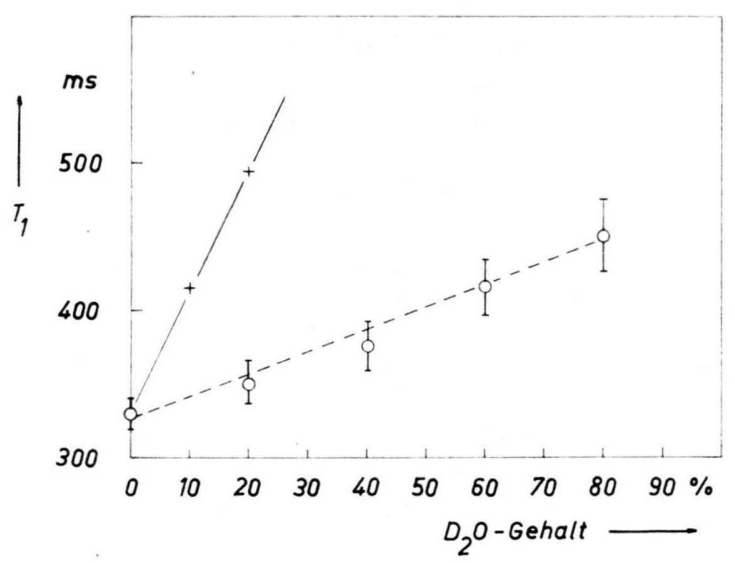

Abb. 3. Abhängigkeit der longitudinalen Protonenrelaxationszeit vom Mischungsverhältnis bei der Adsorption des Wassers an $\mathrm{SiO}_{2}$ II $\left(21^{\circ} \mathrm{C}, \nu=16 \mathrm{MHz}, 600 \mathrm{mg} / \mathrm{g} \mathrm{SiO}_{2}\right.$ II) : $\mathrm{O}=$ expt. Werte, $+=$ berech. nach Gl. (1).

10 H. Sprinz, Z. Naturforschg. 19 a, 1243 [1964]. 


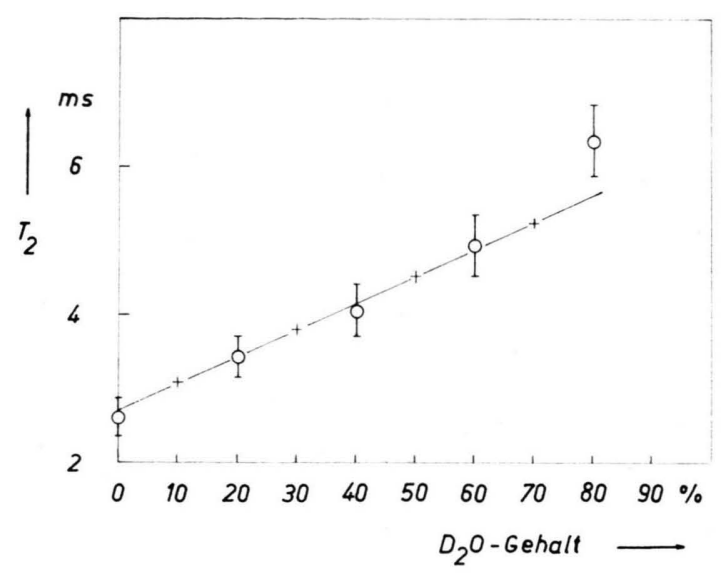

Abb. 4. Abhängigkeit der transversalen Relaxationszeit vom Mischungsverhältnis bei der Adsorption an $\mathrm{SiO}_{2}$ II (vgl. Abb. 3) : $\quad 0=$ expt. Werte, $\quad+=$ berech. nach Gl. (2).

ständen empfindlicher auf paramagnetische Zentren reagiert als die elektronenparamagnetische Resonanz selbst. Völlig andere Resultate für die Protonenrelaxationszeiten $T_{1}$ und $T_{2}$ von $\mathrm{H}_{2} \mathrm{O}-\mathrm{D}_{2} \mathrm{O}$-Gemischen ergeben sich bei Adsorption am $\mathrm{SiO}_{2}$ II, wie man aus den Abb. 3 und 4 erkennt. Der Anstieg der Geraden der $T_{2}$-Werte in Abhängigkeit vom Mischungsverhältnis $\beta$ stimmt mit der Gl. (2) überein. Die Zunahme der $T_{1^{-}}$

11 A. Odajima, Suppl. Progr. Theoret. Phys. (Kyoto) 10, 142 [1959].
Werte ist aber geringer als nach Gl. (1) erwartet wird. Außerdem ist, wie man aus der Tab. 2 erkennt, das Verhältnis $\left(T_{1} / T_{2}\right)$ expt. sogar größer als bei der Annahme der Proton-Proton-Wechselwirkung und einer isotropen Bewegung mit einer Korrelationszeit $\tau_{\mathrm{c}}$. Diese Vergrößerung des $T_{1} / T_{2}$-Verhältnisses kann z. B. dann auftreten, wenn es eine Verteilung von Korrelationszeiten in der Probe gibt ${ }^{11,12}$. Zur Klärung dieses Sachverhaltes und der noch immer zu geringen $\mathrm{Zu}$ nahme der $T_{1}$-Werte mit der Verdünnung sind noch weitere Untersuchungen im Gange.

\begin{tabular}{|c|c|c|c|c|}
\hline System & Zitat & $\begin{array}{l}T_{1} / T_{2} \\
\text { expt. }\end{array}$ & $\begin{array}{c}T_{1} / T_{2} \\
\text { berechn. }\end{array}$ & $\begin{array}{c}\tau_{\mathrm{c}} \text { aus } T_{2} \\
\text { berechn. }\end{array}$ \\
\hline $\mathrm{H}_{2} \mathrm{O}-\mathrm{Al}_{2} \mathrm{O}_{3}$ & $2, *$ & $12^{\circ}$ & 100 & $10^{-7} \mathrm{~s}$ \\
\hline $\begin{array}{l}\mathrm{H}_{2} \mathrm{O}-\mathrm{SiO}_{2} * * \\
\mathrm{H}_{2} \mathrm{O}-\mathrm{SiO}_{2} \mathrm{I} \\
\mathrm{H}_{2} \mathrm{O}-\mathrm{SiO}_{2} \mathrm{II}\end{array}$ & $\begin{array}{l}3 \\
* \\
*\end{array}$ & $\begin{array}{c}2 \cdot 10^{2} \\
5 \\
170\end{array}$ & $\begin{array}{l}2 \cdot 10^{3} \\
3 \dot{4} \\
25\end{array}$ & $\begin{array}{r}3,5 \cdot 10^{-7} \mathrm{~s} \\
6 \cdot 10^{-8} \mathrm{~s} \\
5 \cdot 10^{-8} \mathrm{~s}\end{array}$ \\
\hline
\end{tabular}

* Eigene Messungen.

* Diese Absolutwerte von $\left(T_{1} / T_{2}\right)$ expt. sind mit den anderen nicht vergleichbar, da sie einer geringeren Adsorbatmenge $(\Theta=1 / 3)$ entsprechen.

Tab. 2. $T_{1} / T_{2}$-Verhältnis bei einer Monoschichtbeladung $(\Theta=1)$ von Wasser an verschiedenen Adsorbentien.

Herrn Prof. Dr. Pfrifer danke ich herzlich für die Anregung zu dieser Arbeit und für viele wertvolle Hinweise. Herrn Dr. WINKLER danke ich für Diskussionen.

12 H. A. Resing, J. Chem. Phys. 43, 669 [1965].

\section{BERICHTIGUNGEN}

Zu A. Müller und B. Krebs, Infrarotspektren von Ammonium- und Thallium(I)-pertechnetat, Z. Naturforschg. 20 a, 967 [1965].

In der Überschrift muß es statt „Pertechnat“ heißen: „Pertechnetat“.

Auf Seite 967 (linke Spalte) muß statt „Nujol-Suspensionen und KBr-Preßlinge“ nur „Nujol-Suspensionen“, auf Seite 968 (linke Spalte, 5. Zeile von unten) statt „ $\nu^{2} / v^{4}$ “ ${ } \nu_{2} / \nu_{4}$ " und in Tabelle 1 für $\mathrm{TlTcO}_{4}{ }{ }_{3}=900$ (Sch), 875 (st), 860 (Sch) “ statt „, $v_{3}=918$ (Sch), 905 (Sch), 880 (st) “ stehen.

Vgl. eine ausführliche Arbeit über IR-Spektren kristalliner Permanganate, Pertechnetate und Perrhenate: A. MülLer und B. Krebs, Z. Naturforschg. 21 b, 3 [1966].

Zu B. Krebs und A. Müller, Kraftkonstanten des Trithiocarbonat-Ions, Z. Naturforschg. $20 \mathrm{a}, 1124$ [1965].

Auf Seite 1126 (linke Spalte oben) sind $f_{r a}$ und $f_{r a}^{\prime}$ zu vertauschen.

Auf Seite 1127 (linke Spalte, 3. Zeile von unten) muß es statt „Atomgewicht“ heißen: "Ordnungszahl“.

Zu B. KоскеL, Eine Berechnung des Methanmoleküls II, Band 20 a, 1472 [1965].

Auf S. 1475, rechte Spalte, Abschnitt III, muß der Schlußsatz lauten: „Man findet die Werte der Tab. 1.“ 